\title{
Frequency synchronization of single flux quantum oscillators
}

\author{
Yuki Yamanashi ${ }^{1,2, *}$, Ryo Kinoshita ${ }^{1}$ and Nobuyuki Yoshikawa ${ }^{1,2}$ \\ ${ }^{1}$ Department of Electrical and Computer Engineering, Yokohama National University, 79-5 Tokiwadai, \\ Hodogaya, Yokohama 240-8501, Japan \\ ${ }^{2}$ Institute of Advanced Sciences, Yokohama National University, 79-5 Tokiwadai, Hodogaya, Yokohama \\ 240-8501, Japan \\ E-mail: yamanashi-yuki-kr@ynu.ac.jp
}

Received 21 May 2021, revised 4 August 2021

Accepted for publication 13 August 2021

Published 1 September 2021

\begin{abstract}
We demonstrate the frequency synchronization of multiple single-flux quantum (SFQ) oscillators with different oscillation frequencies. To synchronize these SFQ oscillators, a common constant bias current is supplied to the SFQ oscillators without any bias resistors. When an SFQ oscillator oscillates at a frequency of $f$, the average voltage across the Josephson junction comprising the SFQ oscillator is $f \Phi_{0}$, where $\Phi_{0}$ is the flux quantum in the superconductor. The bias currents supplied to the SFQ oscillators are redistributed to eliminate the average voltage difference output from the SFQ oscillators. As a result, the oscillation frequencies of all the SFQ oscillators are synchronized. Simulation results indicate that SFQ oscillators with an oscillation frequency difference of more than $50 \mathrm{GHz}$ can be synchronized. We experimentally demonstrate the frequency synchronization of two SFQ oscillators composed of circular Josephson transmission lines. Frequency synchronization is expected to contribute toward the development of a low-power stable clock source stabilizing SFQ circuit operation.
\end{abstract}

Keywords: SFQ circuit, synchronization, clock generator, Josephson junction

(Some figures may appear in colour only in the online journal)

\section{Introduction}

Owing to their low-power and high-speed operation, superconducting single-flux quantum (SFQ) circuits $[1,2]$ show potential for use in next-generation integrated circuit technology in the post-Moore era. As conventional SFQ logic gates possess the latching function, synchronized outputs from the logic gates are obtained using clock inputs. Thus, the generation of a high-frequency SFQ clock pulse train is essential for the high-speed operation of SFQ circuits.

\footnotetext{
* Author to whom any correspondence should be addressed.
}

Original Content from this work may be used under the BY
further distribution of this work must maintain attribution to the author(s) and the title of the work, journal citation and DOI.
To generate high-frequency SFQ pulse trains, SFQ oscillators have been studied as on-chip clock generators (CGs) [1,3-6]. On-chip SFQ CGs have been widely utilized in onchip high-speed tests involving the SFQ logic circuits [7, 8] and for bit-shifts in the SFQ arithmetic logic units (ALUs) [9]. In certain applications, the stability of the oscillation frequency of the SFQ oscillators is important. For instance, in SFQ-based analog-to-digital converters $[10,11]$ and timeto-digital converters $[12,13]$, the accuracy of conversion is influenced by the frequency stability of the SFQ clock pulse trains. Furthermore, in random number generation using SFQ circuits, the quality of the generated random number train depends on the frequency stability of the input SFQ pulse train [14-16]. There are many reports on the applications of multiple on-chip CGs to large-scale SFQ circuit systems such as asynchronous handshaking systems $[17,18]$, the SFQ microprocessors [19, 20], and the ERSFQ ALU [21]. In a large-scale SFQ circuit system employing a multichip module [22, 23], it 
is reasonable to implement a clock generation circuit on each chip. However, in such systems, the oscillation frequency of all the SFQ CGs must be identical in order to synchronize the entire system.

In this study, we investigate the frequency synchronization of multiple SFQ oscillators for the generation of stable SFQ clock pulse trains. Herein, a detailed circuit analysis and the experimental demonstration of the frequency synchronization of SFQ oscillators are presented.

\section{Analysis of frequency synchronization}

In this section, we describe the principle of the frequency synchronization of SFQ oscillators and the results of the circuit analysis based on analog circuit simulations. Figure 1 shows the equivalent circuit of the SFQ oscillator under analysis. The analyzed SFQ oscillator comprises a circular Josephson transmission line. To simplify the analysis, we employ a simple and symmetric circuit structure. A constant bias current, $I_{\mathrm{b}}$, is injected into the SFQ oscillator without the use of on-chip bias resistors; by contrast, in the conventional SFQ circuit, the bias currents are supplied via on-chip bias resistors. The SFQ oscillator initiates the oscillation by supplying the appropriate input current $I_{\text {in }}$. When the SFQ oscillator is oscillating at a frequency of $f$, an average voltage of $f \Phi_{0}$ is generated across the Josephson junctions, where $\Phi_{0}$ is the flux quantum in a superconductor.

Figure 2 shows the principle for the frequency synchronization of the SFQ oscillators. In this circuit, two SFQ oscillators (CG1 and CG2) are current-biased by the common constant bias current of $2 I_{\mathrm{b}}$. Both CG1 and CG2 have the same circuit structure and parameters as shown in figure 1, except for the inductance comprising the circular Josephson transmission line. The values of the inductance $L$ of CG1 and CG2 are 4.2 and $3.1 \mathrm{pH}$, respectively. Assuming the use of the AIST $2.5 \mathrm{kA}$ $\mathrm{cm}^{-2} \mathrm{Nb}$ standard process (AIST-STP2) [27, 28], the oscillating frequencies of CG1 and CG2 are 65.8 and $76.6 \mathrm{GHz}$, respectively, when the designed bias current $I_{\mathrm{b}}(=600 \mu \mathrm{A})$ is supplied.

Immediately after oscillation commences, the supplied constant current is divided equally and the current $I_{\mathrm{b}}$ is supplied to CG1 and CG2, as shown in figure 2(a). However, considering the difference between the generated average voltages of CG1 and CG2, the bias currents supplied to CG1 and CG2 are redistributed in order to eliminate this voltage difference $[29,30]$. Therefore, the oscillation frequencies of both CG1 and CG2 are synchronized under the steady state, as shown in figure 2(b). The new concept in this study is the application of the current redistribution in the SFQ circuit to realizing the low-power and stable SFQ oscillators. It should be noted that the static power consumption of SFQ oscillators can be completely eliminated by using frequency synchronization because bias resistors are not used. Therefore, we could build low-power and stable SFQ oscillators using the frequency synchronization.

We simulated the circuit shown in figures 1 and 2 by using the Josephson integrated circuit simulator (JSIM) [31].

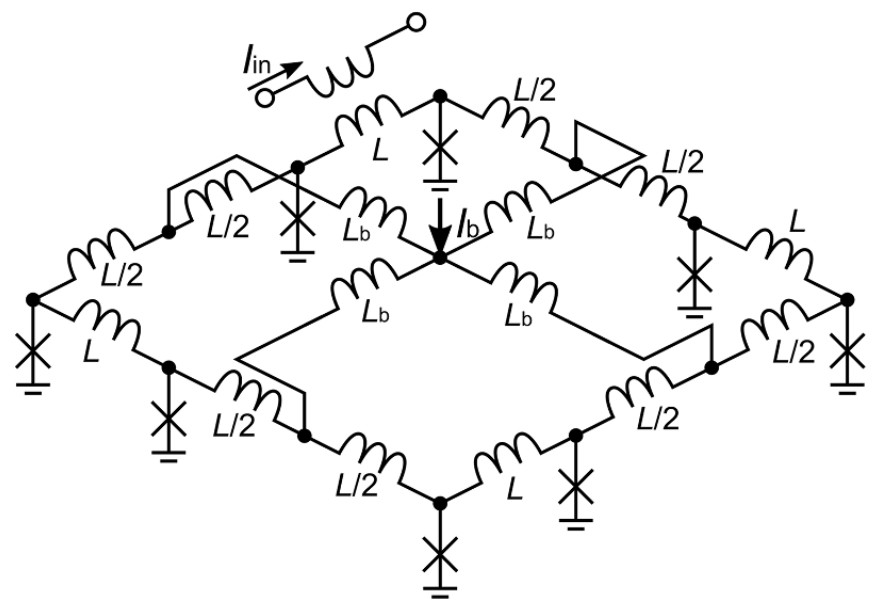

Figure 1. Equivalent circuit of the SFQ oscillator. The cross symbols in the figure represent Josephson junctions. The critical currents of all the Josephson junctions are $100 \mu \mathrm{A} ; L_{\mathrm{b}}=25.0 \mathrm{pH}$, and $I_{\mathrm{b}}=600 \mu \mathrm{A}$. $I_{\text {in }}$ is used to inject the flux quantum into the SFQ oscillator and start oscillation. The McCumber-Stewart parameter $[24,25]$ for all the Josephson junctions is 0.89 , which is the standard value in the CONNECT cell library [26].

(a) Immediately after oscillation commences

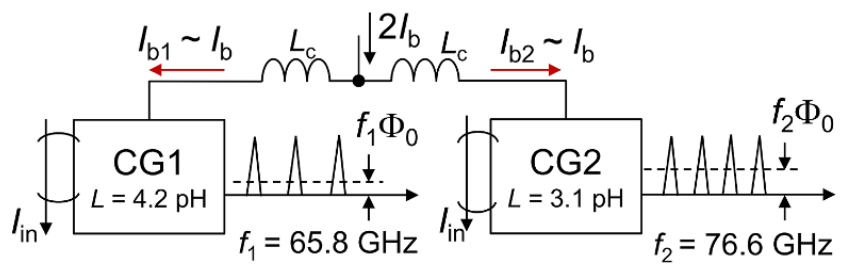

(b) Steady sate

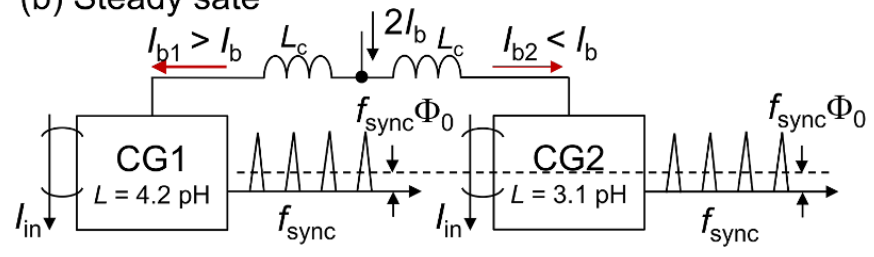

Figure 2. Principle for the frequency synchronization of SFQ oscillators. The bias currents are supplied to CG1 and CG2 via $L_{\mathrm{c}}$. The SFQ oscillators, i.e. CG1 and CG2, are the same as those in figure 1.

$L_{\mathrm{c}}=100 \mathrm{pH}$ was assumed in the simulation. In the circuit simulation, the input current $I_{\text {in }}$ of $634 \mu \mathrm{A}$, which induces the injection of $\Phi_{0}$ in the SFQ oscillators, is simultaneously supplied to both CG1 and CG2. The oscillations of both CG1 and $\mathrm{CG} 2$ commence at $100 \mathrm{ps}$. Figure 3 shows the results of the transient analysis of the voltages across one of the Josephson junctions in CG1 and CG2. Figure 4 shows the transition of the oscillation frequencies of the SFQ oscillators and the bias currents supplied to CG1 and CG2.

Immediately after the oscillation commences (i.e. at $100 \mathrm{ps}$ in the simulation), CG1 and CG2 oscillate at frequencies of 65.8 and $76.3 \mathrm{GHz}$, respectively, which are almost identical to the individual oscillation frequencies of CG1 and CG2 when they are biased at $I_{\mathrm{b}}=600 \mu \mathrm{A}$. After oscillation commences, the bias currents supplied to $\mathrm{CG} 1$ and $\mathrm{CG} 2$ (i.e. $I_{\mathrm{b} 1}$ 


\section{(a) Immediately after oscillation commences}

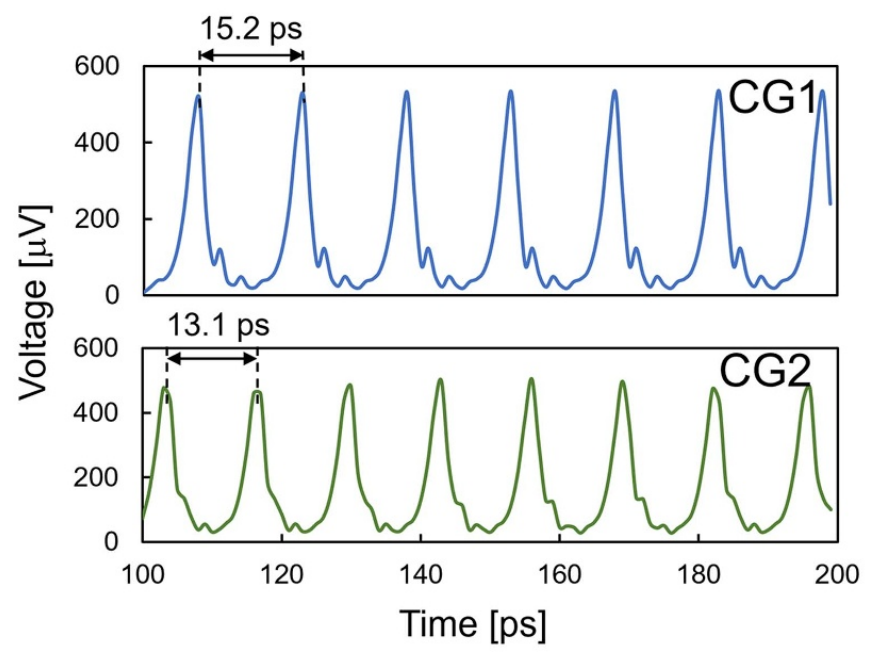

(b) Steady sate

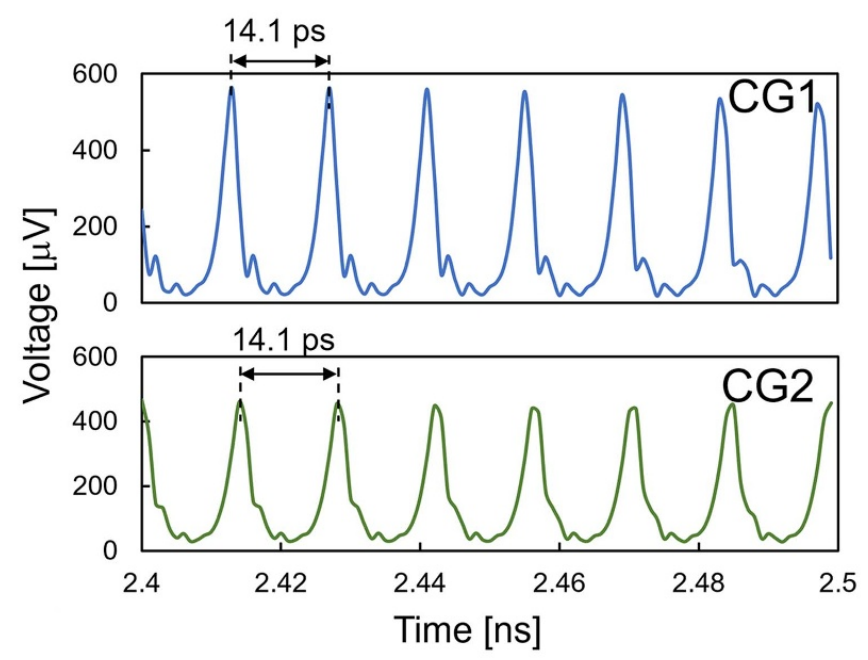

Figure 3. Transient analysis results for the frequency synchronization of CG1 and CG2.

(a)

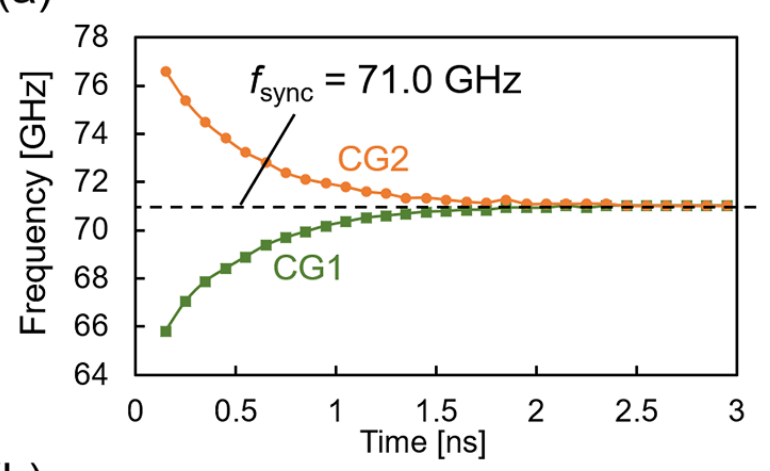

(b)

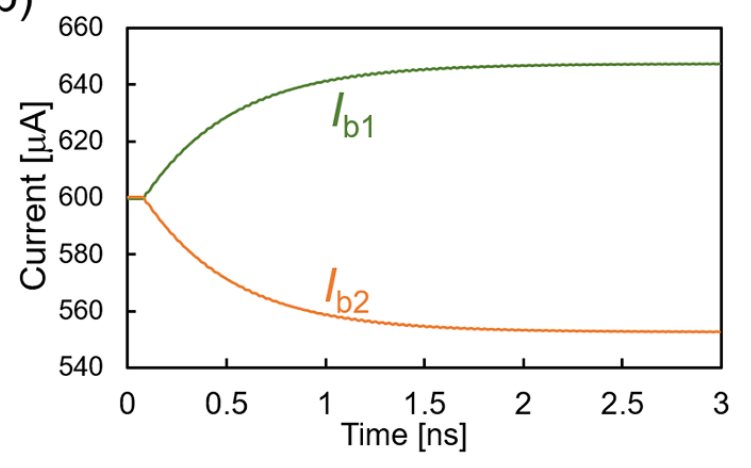

Figure 4. Simulated transition of (a) oscillating frequencies of CG1 and CG2 and (b) bias currents supplied to CG1 and CG2. $L_{\mathrm{c}}=100$ $\mathrm{pH}$. The oscillations of both CG1 and CG2 commence at $100 \mathrm{ps}$.

and $I_{\mathrm{b} 2}$, respectively) are redistributed to eliminate the generated average voltage difference between CG1 and CG2, as shown in figure 4(b). Approximately $2.3 \mathrm{~ns}$ after the commencement of oscillation, $I_{\mathrm{b} 1}$ and $I_{\mathrm{b} 2}$ are saturated, and the oscillation frequencies of both CG1 and CG2 are synchronized to $71.0 \mathrm{GHz}$.
The time required for frequency synchronization is determined by the $L / R$ time constant of the bias circuits of the SFQ oscillators. In the analyzed SFQ oscillators, the resistance of the bias circuit is approximately equal to the total resistance of eight shunt resistors of Josephson junctions connected in parallel. The inductance of the bias circuit is determined by $L_{\mathrm{b}}$ and $L_{\mathrm{c}}$. The time required for frequency synchronization could be reduced by reducing $L_{\mathrm{b}}$ and $L_{\mathrm{c}}$. However, the stability of the oscillating frequency after frequency synchronization deteriorates when the inductance of the bias circuit is reduced. Appropriate choice of $L_{\mathrm{b}}$ and $L_{\mathrm{c}}$ is important to obtain both fast frequency synchronization and the stable synchronized frequency.

Furthermore, we evaluate how the synchronization frequency $\left(f_{\text {sync }}\right)$ is determined by the individual oscillating frequencies of CG1 and CG2. In this analysis, the oscillation frequency of CG1 $\left(f_{1}\right)$ is fixed to $65.8 \mathrm{GHz}$ by setting $L$ in CG1 to $4.2 \mathrm{pH}$. Moreover, $f_{2}$ is adjusted by varying the value of $L$ in $\mathrm{CG} 2$. Figure 5 shows the simulated dependence of the synchronization frequency on the oscillation frequency of CG2 $\left(f_{2}\right)$. In this manner, we confirm that the frequency synchronization occurs when $f_{2}$ ranges from 4.9 to $127.0 \mathrm{GHz}$. As shown in figure 5 , when $f_{2}$ is close to $f_{1}, f_{\text {sync }}$ is close to $\left(f_{1}+f_{2}\right) / 2$. This result indicates that the dependence of the oscillation frequencies of the SFQ oscillators on the supplied bias current can be regarded as linear near the center of the operating bias region. However, when the difference between $f_{1}$ and $f_{2}$ is large, $f_{\text {sync }}$ approaches $f_{1}$. This result indicates that the operating point of CG1 is automatically shifted toward the center of the operating margin. This phenomenon could possibly stabilize the operation of SFQ circuits that are driven by non-optimal bias currents. The frequency synchronization can be applied to any on-chip SFQ clock source, such as single Josephson junction-based SFQ oscillators [32, 33] and long Josephson junction oscillators $[34,35]$. 


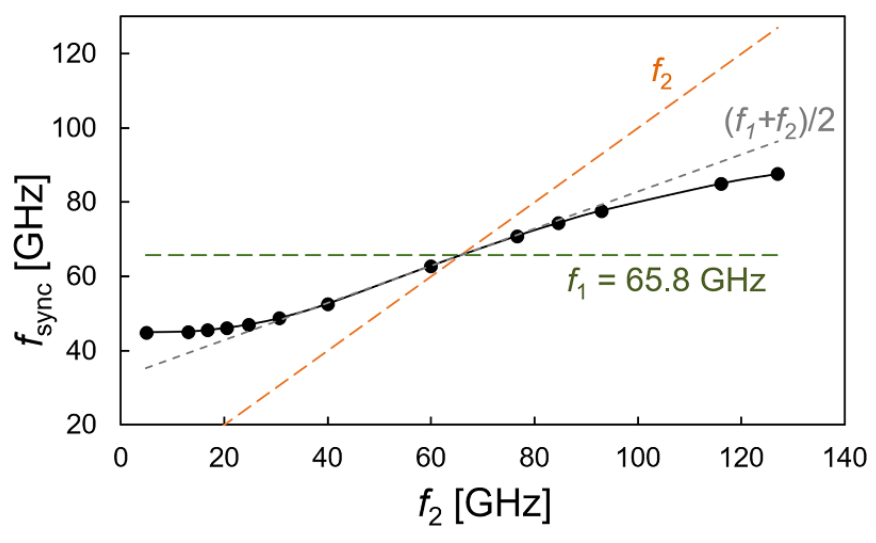

Figure 5. Simulated dependence of the synchronization frequency on the oscillating frequency of CG2. The dots represent the simulated $f_{\text {sync. }}$. The dashed lines are inserted to discuss the simulation result. The oscillation frequency of CG1 $\left(f_{1}\right)$ is fixed at $65.8 \mathrm{GHz}$.

\section{Experimental}

We designed test circuits using the AIST $10 \mathrm{kA} \mathrm{cm}^{-2} \mathrm{Nb}$ high-speed standard process (AIST-HSTP) $[36,37]$ to demonstrate the frequency synchronization of two SFQ oscillators. We used a three-dimensional inductance extraction tool InductEX [38] was used for precise inductance design. Figure 6 shows the photomicrograph of the test circuit. For this test circuit, the equivalent circuit and the circuit parameters, except for the critical current density, are identical to those discussed in the previous section. Individual test circuits where either CG1 or CG2 is simply biased by $I_{\mathrm{b}}$ are also prepared. By comparing the oscillation frequencies in these three test circuits, we demonstrate the frequency synchronization of CG1 and CG2.

All the test circuits are cooled to $4.2 \mathrm{~K}$ in a liquid helium bath and then analyzed. We measured the oscillating frequency $(f)$ of each SFQ oscillator by measuring the average output voltages across the Josephson junctions, where terminals $V_{\text {monitor1 }}$ and $V_{\text {monitor2 }}$ are attached; the oscillating frequency is calculated as follows:

$$
f=\frac{V}{\Phi_{0}},
$$

where $V$ is the measured average voltage [39]. The average voltages output from the SFQ oscillators were measured using a nanovoltmeter (Keithley 2182A). When $I_{\text {in }}$ of $700 \mu \mathrm{A}$ was supplied to the test circuit shown in figure 6 , we obtained the same voltages from both $V_{\text {monitor1 }}$ and $V_{\text {monitor2. This indic- }}$ ates that both CG1 and CG2 were oscillating at the same frequency.

Figure 7 shows the measured dependences of the oscillation frequencies of the three test circuits on the supplied bias current. The measured oscillation frequency of the test circuit in figure $6, f_{\text {sync }}$, lies between the individual oscillation frequencies of CG1 and CG2 for all the bias regions. This also indicates the frequency synchronization of CG1 and CG2.

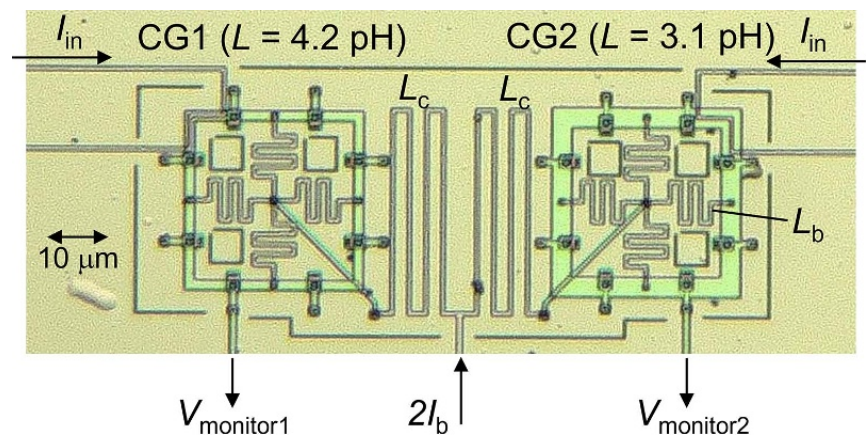

Figure 6. Photomicrograph of the test circuit used to demonstrate frequency synchronization. $V_{\text {monitor1 }}$ and $V_{\text {monitor2 }}$ are terminals for measuring the average voltage of the Josephson junctions in the corresponding SFQ oscillators. The Josephson junctions are shunted using $11.32 \Omega$ in order to set the McCumber-Stewart parameter to 2. $L_{\mathrm{c}}=100 \mathrm{pH}$ and $L_{\mathrm{b}}=25.0 \mathrm{pH}$.

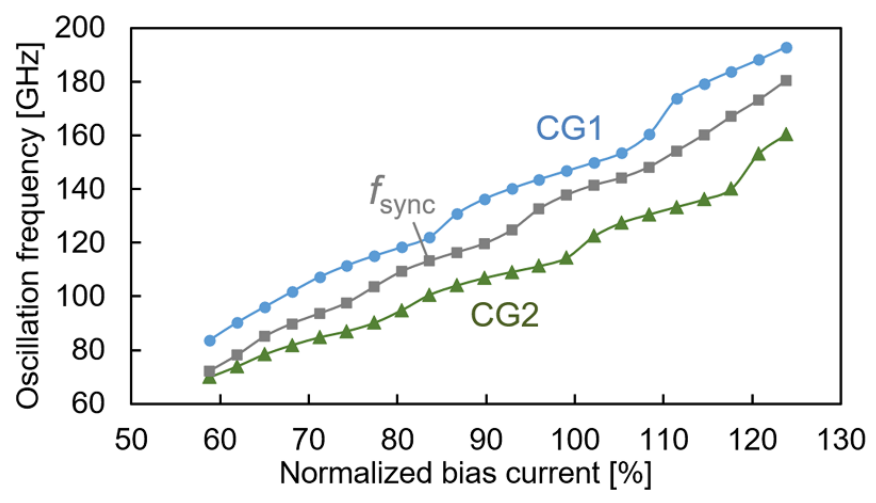

Figure 7. Measured dependences of the oscillation frequencies of test circuits. $f_{\text {sync }}$ is the measured result for the test circuit in figure 6. CG1 and CG2 refer to the individual test results of CG1 and $\mathrm{CG} 2$, respectively.

\section{Conclusion}

We successfully realized the frequency synchronization of SFQ oscillators with different oscillation frequencies by using the common current biasing. Results of the analog circuit simulations indicated that the current redistribution, which nullified the average voltage difference among multiple SFQ oscillators, induced the frequency synchronization of the SFQ oscillators. Furthermore, we experimentally observed the frequency synchronization in all bias regions. We believe that frequency synchronization can facilitate the stabilization of the high-speed operation of SFQ circuits.

\section{Data availability statement}

The data that support the findings of this study are available upon reasonable request from the authors.

\section{Acknowledgments}

This work was supported by JSPS KAKENHI Grant Numbers JP18K04280, 19H01945 and JP19H05614. The circuits were 
fabricated in the clean room for analog-digital superconductivity (CRAVITY) of National Institute of Advanced Industrial Science and Technology (AIST) with the high-speed standard process (HSTP).

\section{ORCID iD}

Yuki Yamanashi (D) https://orcid.org/0000-0003-4840-7611

\section{References}

[1] Likharev K K and Semenov V K 1991 RSFQ logic/memory family: a new Josephson-junction technology for sub-terahertz-clock-digital systems IEEE Trans. Appl. Supercond. 13-28

[2] Nakajima K, Mizusawa H, Sugahara H and Sawada Y 1991 Phase mode Josephson computer system IEEE Trans. Appl. Supercond. $129-36$

[3] Lin J C and Semenov V K 1995 Timing circuits for RSFQ digital systems IEEE Trans. Appl. Supercond. 5 3472-7

[4] Mancini C A and Bocko M F 1999 Phase-locked operation of RSFQ ring oscillators Supercond. Sci. Technol. 12 789-91

[5] Brock D K 2001 RSFQ technology: circuits and systems Int. J. High Speed Electron. Syst. 11 307-62

[6] Katsuno H, Nagano T, Nakayama K and Yoshida J 2003 HTS-SFQ ring oscillator circuit fabricated with a novel multilayer structure Phys. C 392-396 1433-40

[7] Deng Z J, Yoshikawa N, Whiteley S R and Van Duzer T 1997 Data-driven self-timed RSFQ digital integrated circuit and system IEEE Trans. Appl. Supercond. 7 3634-7

[8] Kirichenko A F, Mukhanov O A and Ryzhikh A I 1997 Advanced on-chip test technology for RSFQ circuits IEEE Trans. Appl. Supercond. 7 3438-41

[9] Kirichenko A F, Kamkar M Y, Walter J and Vernik I V 2019 ERSFQ 8-bit parallel binary shifter for energy-efficient superconducting CPU IEEE Trans. Appl. Supercond. 291302704

[10] Mukhanov O A, Gupta D, Kadin A M and Semenov V J 2004 Superconductor analog-to-digital converters Proc. IEEE 92 1564-84

[11] Suzuki H, Oikawa M, Nishii K, Ishihara K, Fujiwara K, Maruyama M and Hidaka M 2011 Design and demonstration of a 5-bit flash-type SFQ A/D converter integrated with error correction and interleaving circuits IEEE Trans. Appl. Supercond. 21 671-6

[12] Sano K, Yamanashi Y and Yoshikawa N 2014 Design and demonstration of a single-flux-quantum multi-stop time-to-digital converter for time-of-flight mass spectrometry IEICE Trans. Electron. E97.C 182-7

[13] Tomitsuka Y, Yamanashi Y, Zen N, Ohkubo M and Yoshikawa N 2019 Demonstration of picosecond time resolution in double-oscillator time-to-digital converter using single-flux-quantum circuits IEEE Trans. Appl. Supercond. 291301505

[14] Yamanashi Y and Yoshikawa N 2009 Superconductive random number generator using thermal noises in SFQ circuits IEEE Trans. Appl. Supercond. $19630-3$

[15] Sugiura T, Yamanashi Y and Yoshikawa N 2011 Demonstration of $30 \mathrm{Gbit} / \mathrm{s}$ generation of superconductive true random number generator IEEE Trans. Appl. Supercond. 21 843-6
[16] Onomi T and Mizugaki Y 2020 Hardware random number generator using Josephson oscillation and SFQ logic circuits IEEE Trans. Appl. Supercond. 301301305

[17] Ito M, Kawasaki K, Yoshikawa N, Fujimaki A, Terai H and Yorozu S $200520 \mathrm{GHz}$ operation of bit-serial handshaking systems using asynchronous SFQ logic circuits IEEE Trans. Appl. Supercond. 15 255-8

[18] Nobumori Y, Nishigai T, Nakamiya K, Yoshikawa N, Fujimaki A, Terai H and Yorozu S 2007 Design and implementation of a fully asynchronous SFQ microprocessor: SCRAM2 IEEE Trans. Appl. Supercond. 17 478-81

[19] Tanaka M et al 2006 Design of a pipelined 8-bit-serial single-flux-quantum microprocessor with multiple ALUs Supercond. Sci. Technol. 19 S344-9

[20] Yamanashi Y et al 2007 Design and implementation of a pipelined bit-serial SFQ microprocessor, CORE1 $\beta$ IEEE Trans. Appl. Supercond. 17 474-7

[21] Kirichenko A F, Vernik I V, Kamkar M Y, Walter J, Miller M, Albu L R and Mukhanov O A 2019 ERSFQ 8-Bit parallel arithmetic logic unit IEEE Trans. Appl. Supercond. 291302407

[22] Hashimoto Y, Yorozu S and Miyazaki T 2005 Transmission of single-flux-quantum pulse between superconductor chips Appl. Phys. Lett. 86072502

[23] Kaplan S B, Dotsenko V and Tolpygo D 2007 High-speed experimental results for an adhesive-bonded superconducting multi-chip module IEEE Trans. Appl. Supercond. 17 971-4

[24] McCumber D E 1968 Effect of ac impedance on dc voltage-current characteristics of superconductor weak-link junctions J. Appl. Phys. 39 3113-18

[25] Stewart W C 1968 Current-voltage characteristics of Josephson junctions Appl. Phys. Lett. 12 277-80

[26] Yorozu S, Kameda Y, Terai H, Fujimaki A, Yamada T and Tahara S 2002 A single flux quantum standard logic cell library Phys. C 378-381 1471-4

[27] Nagasawa S, Hashimoto Y, Numata H and Tahara S 1995 A $380 \mathrm{ps}, 9.5 \mathrm{~mW}$ Josephson 4-kbit RAM operated at a high bit yield IEEE Trans. Appl. Supercond. $52447-52$

[28] Hidaka M, Nagasawa S, Satoh T, Hinode K and Kitagawa Y 2006 Current status and future prospect of the Nb-based fabrication process for single flux quantum circuits Supercond. Sci. Technol. 19 S138-42

[29] Kirichenko D E, Sarwana S and Kirichenko A F 2011 Zero static power dissipation biasing of RSFQ circuits IEEE Trans. Appl. Supercond. 21 760-9

[30] Mukhanov O A 2011 Energy-efficient single flux quantum technology IEEE Trans. Appl. Supercond. 21 769-70

[31] Fang E S and Van Duzer T 1989 A Josephson integrated circuit simulator (JSIM) for su-perconductive electronics application Extended Abstracts Int. Supercond. Electron. Conf. pp 407-10

[32] Chen W, Rylyakov A V, Patel V, Lukens J E and Likharev K K 1998 Superconductor digital frequency divider operating up to $750 \mathrm{GHz}$ App. Phys. Lett. 73 2817-19

[33] Bunyk P I, Oliva A, Semenov V K, Bhushan M, Likharev K K, Lukens J E, Ketchen M B and Mallison W H 1995 High-speed singleflux-quantum circuit using planarized niobium-trilayer Josephson junction technology App. Phys. Lett. 66 646-8

[34] Gupta D and Zhang Y 2000 On-chip clock technology for ultrafast digital superconducting electronics App. Phys. Lett. 76 3819-21 
[35] Vernik I V and Gupta D 2003 Two-phase 50 GHz on-chip long Josephson junction clock source IEEE Trans. Appl. Supercond. 13 587-90

[36] Hidaka M and Nagasawa S 2021 Fabrication process for superconducting digital circuits IEICE Trans. Electron. to be published (https://doi.org/10.1587/transele. 2020SUI0002)

[37] Takeuchi N, Nagasawa S, China F, Ando T, Hidaka M, Yamanashi Y and Yoshikawa N 2017 Adiabatic quantum-flux-parametron cell library designed using a 10
$\mathrm{kA} \mathrm{cm}^{-2}$ niobium fabrication process Supercond. Sci. Technol. 30035002

[38] Fourie C J, Wetzstein O, Ortlepp T and Kunert J 2011 Three-dimensional multi-terminal superconductive integrated circuit inductance extraction Supercond. Sci. Technol. 24125015

[39] Iwasaki S, Tanaka M, Irie N, Fujimaki A, Yoshikawa N, Terai H and Yorozu S 2007 Quantitative evaluation of delay time in the single-flux-quantum circuit Phys. C 463-465 1068-71 